\title{
Mathematicians' Example-Related Activity when Exploring and Proving Conjectures
}

\author{
Elise Lockwood ${ }^{1}$ • Amy B. Ellis ${ }^{2}$ - Alison G. Lynch $^{3}$
}

Published online: 25 February 2016

(C) Springer International Publishing Switzerland 2016

\begin{abstract}
Examples can play a critical role in the exploration of conjectures and in the subsequent development of proofs. Although proof has been an object of extensive study, there is more to learn about the precise ways in which mathematicians leverage examples as they formulate proofs. In this paper, we present results from surveys and interviews with mathematicians that targets the role of examples in mathematicians' proof-related activity. Their responses shed light on specific example-related activity (including strategic example selection and use) and on the overarching ways in which they engage in such activity (including a focus on generalization and metacognition). We share illustrative excerpts from the surveys and interviews and discuss educational implications of the results.
\end{abstract}

Keywords Examples $\cdot$ Proof $\cdot$ Mathematicians

\section{Introduction}

Proof is a perennial topic in mathematics education, and although many cases have been made for its importance across age levels (e.g., Ball et al. 2002; Knuth 2002a, b;

Elise Lockwood

elise314@gmail.com

Amy B. Ellis

aellis1@education.wisc.edu

Alison G. Lynch

alynch@csumb.edu

1 Department of Mathematics, Oregon State University, 064 Kidder Hall, Corvallis, OR 97331, USA

2 University of Wisconsin - Madison, 476D Teacher Education Building, 225N Mills St., Madison, WI 53706, USA

3 Department of Mathematics and Statistics, California State University, Monterey Bay, 100 Campus Center, Seaside, CA 93955, USA 
Sowder and Harel 1998; Yackel and Hanna 2003; CCSS 2010; NCTM 2000), students' difficulties with proof persist (Kloosterman and Lester 2004). Some researchers have suggested that students' struggles with understanding the nature of evidence and justification may be due, in part, to their views concerning the role and status of examples. In particular, students tend to be overly reliant on examples and often infer that a (universal) mathematical statement is true on the basis of checking a number of examples that satisfy the statement (e.g., Healy and Hoyles 2000; Knuth et al. 2009; Porteous 1990). Much of the current literature on teaching proof in school mathematics underscores the goal of helping students understand the limits of such example-based reasoning (e.g., Harel and Sowder 1998; Stylianides and Stylianides 2009; Zaslavsky et al. 2012) and typically characterizes example-based reasoning strategies as obstacles to overcome.

Although this line of research has yielded important findings, research on mathematicians' abilities to leverage examples to support conjecturing and proving suggests that example-based reasoning strategies should not be positioned only as barriers to negotiate. Indeed, the field may benefit from a deeper understanding of the ways in which those who are adept at proof, such as mathematicians, critically analyze and leverage examples in order to support their proof-related thinking and activity. The goal of this paper is to characterize expert mathematicians' example-related activity in the context of exploring and proving mathematical conjectures. We report on findings that arose during surveys and clinical interviews, examining the nature of mathematicians' example use while investigating conjectures and developing proofs, and we discuss potential implications for the teaching and learning of proof. First, we describe the example-related activities with which mathematicians engaged in the context of proof, which includes descriptions of ways in which mathematicians a) strategically select examples, and b) strategically use examples. Second, we describe the mathematicians' overarching purposeful engagement with such activities, which includes ways in which mathematicians flexibly focus on generalization and demonstrate metacognitive awareness of the role of examples in their overall proving activity.

\section{Theoretical Framework and Relevant Literature}

\section{Defining Key Terms}

We begin by clarifying how we define examples and proof. We follow Bills and Watson (2008) in defining an example broadly, as "any mathematics object from which it is expected to generalize" (p. 78). We also agree with Goldenberg and Mason (2008) who characterize examples as cultural mediating tools between learners and mathematics concepts, theorems, and techniques, noting that examples are a major means of mathematical communication, either with oneself or with others. From this perspective, examples are situated within the learner's understanding; a mathematical object is therefore only an example when the learner perceives it to be an example of something: "The fundamental construct is the act of seeing something as an example of some 'thing'" (Goldenberg and Mason 2008, p. 184, emphasis in the original).

We draw on Harel and Sowder's (1998) definition of proving, which is "the process employed by an individual to remove or create doubts about the truth of an 
observation" (p. 241). Harel and Sowder further distinguish between two kinds of activity associated with proving - ascertaining, which they define as "the process an individual employs to remove her or his own doubts about the truth of an observation" (p. 241), and persuading, which is "the process an individual employs to remove others' doubts about the truth of an observation" (p. 241). We consider proving as encompassing both kinds of activities. ${ }^{1}$

\section{Background Literature on Examples}

It is generally accepted that students' justifications are expected to progress from empirical arguments to proofs (e.g., Simon and Blume 1996). However, in this progression, caution must be made so that students do not view examples as constituting a proof or as being a valid substitution for a proof. There is a trend in the literature toward helping K-12 students and pre-service teachers understand the limitations of examples as a means of justification and thus recognize the need for a proof (e.g., Sowder and Harel 1998; Stylianides and Stylianides 2009; Zaslavsky et al. 2012). There is also growing evidence, however, that some students $d o$ recognize the limitations of examples as sufficient for proof. For instance, Weber (2010) found that undergraduate mathematics majors did not find empirical arguments to meet the standards of proof, and that participants' difficulties with proof did not stem from a propensity to be convinced by empirical arguments. Weber's findings suggest that improving students' comprehension of, and ability to produce, deductive justifications will require going beyond merely helping them see the limitations of empirical arguments. One route of investigation we consider promising is to better understand the ways in which people can actually use examples productively in order to form or make sense of conjectures and to develop general ideas that can support the production of a deductive proof.

Recent years have seen an increased attention on example use in the mathematics education literature. Researchers have identified and categorized types of examples (Buchbinder and Zaslavsky 2009; Mason and Watson 2008; Michener 1978; Tsamir et al. 2008; Zazkis and Leikin 2008), investigated people's thinking with and use of examples (Antonini 2006; Balacheff 1987; Bills and Rowland 1999; Buchbinder and Zaslavsky 2011; Goldenberg and Mason 2008; Watson and Mason 2005; Watson and Shipman 2008; Zodik and Zaslavsky 2008), and studied the relationships between example use and proof (Antonini 2003; Alcock and Inglis 2008; Chazan 1993; Iannone et al. 2011; Pedemonte and Buchbinder 2012; Rowland 2001; Sandefur et al. 2013). This body of research as a whole reveals the richness and variety of the types of examples learners are able to produce, examine, and leverage as they make sense of new ideas, consider and formulate conjectures, and develop deductive arguments.

\section{Literature Framing Our Study}

Research on mathematicians' conjecturing and proving activity suggests that example use plays a critical role both in the development of and exploration of conjectures, as

\footnotetext{
${ }^{1}$ We use inductive and empirical interchangeably to refer to example-based arguments, and we use deductive and proof interchangeably to refer to arguments comprised of a series of logically connected assertions that one makes to justify a mathematical claim.
} 
well as in the subsequent construction of proofs of those conjectures. Epstein and Levy (1995) contend that "Most mathematicians spend a lot of time thinking about and analyzing particular examples," and they go on to note that "It is probably the case that most significant advances in mathematics have arisen from experimentation with examples" (p. 6). Several researchers have accordingly examined various aspects of the interplay between example-based reasoning activities and proof activities among both mathematicians and mathematics students (e.g., Alcock and Inglis 2008; Antonini 2006; Buchbinder and Zaslavsky 2009; Iannone et al. 2011; Harel 2008; Knuth et al. 2009; Weber 2008, 2010; Weber et al. 2014). For instance, Alcock and Inglis (2008) constructed case studies of two participants engaged in example use while evaluating conjectures and constructing proofs. They found that the student who was able to produce robust and logically consistent justifications also made use of a variety of examples for multiple purposes, including developing understanding, inductive testing of conjectures, checking arguments, and generating counterexamples. Other researchers have similarly identified ways in which example use can be an effective tool to support proof construction (e.g., Antonini 2003; Buchbinder and Zaslavsky 2011; Pedemonte and Buchbinder 2012; Rowland 2001) and to determine an argument's validity (Weber 2008). For instance, Antonini (2003) found that the generation of non-examples can lead to indirect argumentation and proof by contraction, and Pedemonte and Buchbinder (2012) found that examples appear to be particularly helpful for proof development when they allow cognitive unity and structural continuity between argumentation and proof.

We build on two existing studies that have categorized example-related activity. First, Antonini (2006) interviewed advanced graduate students, asking them to generate examples with specific mathematical properties. His work yielded an initial categorization scheme for strategies used to produce examples, which include trial and error, transformation, and analysis. Although our study differs in emphasis, Antonini's categorization of advanced graduate students' strategies provides a starting point for developing our themes. He points out that

Further research is also needed to study how the identified strategies are intertwined with processes enacted in different situations where subjects produce examples. We also believe that these strategies may be useful to observe processes of production of examples in tasks involving a careful exploration in order to produce conjectures and proofs (p. 63).

Second, building on Antonini's (2006) framework, Iannone et al. (2011) categorized undergraduate students' strategies in example generation. Iannone et al. found that simply generating examples when asked to do so did not improve participants' abilities to write proofs more than examining provided examples. These findings suggest that example generation alone may not be sufficient to support productive proof activities; rather, it is the ways in which learners can effectively leverage examples that matters in serving as a bridge to appropriate deductive argumentation. Iannone and colleagues' work underscores the complexity of studying examples, and they suggest that "There is clearly a need for further empirical research in this area if we are to determine whether and how example generation tasks can lead to significant learning gains" (p. 11). 
In the study reported in this paper, we carefully examine the example-related activity of mathematicians, whom we take to be experienced provers. Our work extends the body of research by systematically investigating the role of examples for expert mathematicians as they explore and attempt to prove mathematical conjectures. By studying mathematicians' thinking, we seek to better understand how they leverage examples in their conjecturing and proving activities. It is important to note that studies of expert thinking should not be applied uncritically to novice instruction. However, we agree with Weber et al. (2014) that even if there are inconsistencies between mathematicians' practices and what is taught in classrooms,

Recognizing such inconsistencies requires having an accurate understanding of mathematical practice. Even if it is not problematic for students to be ignorant of mathematical practice, it does not follow that it is acceptable for teachers, researchers, or curriculum designers to be similarly unaware (p. 38).

We thus work under the assumption that gaining insights into the ways in which mathematicians productively work with examples can provide us with a better understanding of potential ways to leverage examples for proof.

\section{Methods}

There are two types of data reported in this study: 1) mathematicians' open-ended responses to a large-scale survey, and 2) individual interviews in which mathematicians explored conjectures. These two forms of data are meant to be complementary, and the interviews served to elaborate and refine the initial results from the survey. We describe the methods of the data collection and analysis for each of these forms of data separately.

\section{Surveys}

The survey data presented in this paper are one aspect of a larger survey given to mathematicians. The surveys were sent to the mathematics departments at 39 universities across the United States. The goal was to gather a large number of responses to the broader survey, and so we targeted universities with large mathematics departments. Included in the 39 universities were 16 private universities and 23 public universities, all of which were $\mathrm{PhD}$ granting research universities. Although the survey primarily included questions about typicality and similarity among examples (as part of a separate line of inquiry), the data discussed in this paper consisted of 220 mathematician responses to one open-response prompt: If you sometimes use examples when exploring a new mathematical conjecture, how do you choose the specific examples you select in order to test or explore the conjecture? What explicit strategies or example characteristics, if any, do you use or consider? Approximately $55 \%$ of the mathematicians who filled out the survey were completing $\mathrm{PhD}$ 's in mathematics, $31 \%$ had $\mathrm{PhD}$ 's in mathematics (in a variety of different mathematical areas), and $13 \%$ had advanced degrees in other STEM-related fields; $66 \%$ were male. In total, 291 mathematicians responded to the survey, and 220 of these responded to this prompt. The survey data consist of these expert mathematicians' selfreported responses about their work with examples. 
Analysis of the survey data consisted of examining the data for emergent themes and categories, using the constant comparison method (e.g., Strauss and Corbin 1998). Specifically, two members of the research team independently examined the survey responses with the intent of identifying the various types of examples the mathematicians reported. These codes were informed by initial categorizations (such as in Antonini 2006), and we were aware of the kinds of activity that we might observe (considering work by Epstein and Levy 1995 and Weber 2010). With such previous work in mind we attempted to code the responses according to emergent phenomena that we encountered. We coded both types and uses of examples that mathematicians worked with, as well as their strategies for including examples in their work (such as intentionally building up a progression of examples). After codes for types, uses, and strategies emerged, two members of the research team re-coded all of the responses; any discrepancies were resolved through discussion with the research team. The research team then discussed overall themes that we observed in the survey data.

As an example of the coding process, we consider two survey responses. The first response was, "The examples I try often tend to be of extremal nature (sort of a worst or best case scenario). I also choose examples with which I am already most familiar, since I should be able to resolve more quickly whether the conjecture is true in these cases." This kind of response gave us insight into both the types of examples that mathematicians chose, and also a potential use for a chosen example. This mathematician reported choosing examples of "extremal nature," which we coded as a boundary case because we interpreted the extreme nature of an example to be testing the boundary of a conjecture or situation. The mathematician also reported choosing examples "with which I am already most familiar," which was coded as a familiar/known case. In addition, because the mathematician said "the examples I try" and "I should be able to resolve more quickly whether the conjecture is true," we coded the example use as check the conjecture, by which we mean that examples are used to check or test whether the conjecture is true.

As a comparison, another response was "I first do examples that are easiest to test. If those are consistent with the conjecture, I try more general examples, focusing on those for which the conjecture might fail for." Here again we gain insight into both types and uses of examples. For types, the language "easiest to test" suggests that the mathematician tests examples that are easy to compute, which was a type that emerged repeatedly. In addition, the statement "I try more general examples, focusing on those for which the conjecture might fail for" offers two additional types: general/generic and conjecture-breaking. In terms of uses, the "easiest to test" language suggested a use code of check the conjecture, while the emphasis on examples for which the conjecture might fail led to a use code of break the conjecture, because the mathematician seemed to use examples with the goal of potentially breaking the conjecture. This response also highlights a strategy that we coded, which was a progression of examples: increasing in generality. We coded this strategy because the mathematician articulated a multi-stage progression from easy to more general examples.

\section{Interviews}

After the surveys were collected and analyzed, we conducted two sets of semistructured, task-based interviews. We followed the surveys with interviews for two reasons. First, because the surveys yielded self-reported data, we wanted to see whether 
similar findings emerged as mathematicians actually explored and proved conjectures in practice. Additionally, because the surveys did not allow for interaction or follow-up questions, we wanted to have the opportunity to engage with mathematicians and to ask deeper questions that might better illuminate their thinking and perspectives. The interviews allowed us to investigate the extent to which the reported categories in the framework actually aligned with what mathematicians do in practice. Although the interviews were not completely authentic proving situations for mathematicians, the mathematicians were at least put in situations in which they were asked to prove, not just reflect about proving.

\section{Data Collection}

For the interview recruitment, we sent emails to mathematicians within the surveyed mathematics departments, inviting any interested mathematician to participate. We are not aware of whether or not the mathematicians who were interviewed were also survey respondents (the survey responses were anonymous and we did not gather contact information). In collecting interview data, we first interviewed six mathematicians as they explored and attempted to prove three mathematical conjectures (we will call this IS1, for Interview Set 1), and then we interviewed 13 different mathematicians as they explored one or two tasks (IS2, for Interview Set 2). The rationale for conducting the second set of interviews was to support findings from the surveys and the first set of interviews and to attempt to saturate the data. In addition, after IS1, in which mathematicians were given a limited amount of time to explore three conjectures, we felt more could be learned from giving mathematicians more time to work. We thus structured IS2 to allow for more time on a task, so we might gain new insight into their work when they had fewer time constraints. The three sets of data (the surveys and both sets of interviews) together paint a vivid picture of mathematicians' examplerelated activity as they explore and prove conjectures.

Figure 1 below shows Conjectures 1 through 4, which were used in IS1. Figure 2 shows Tasks 1 and 2, which were used in IS2. Conjectures 1-3 and Task 2 were taken from Putnam exams, Conjecture 4 was adapted from tasks in Alcock and Inglis (2008), and Task 1 was taken from Andreescu et al. (2007). For both interview sets, we chose these problems with two primary criteria in mind. First, the conjectures were accessible to the mathematicians (regardless of their area of expertise) but were not so clearly obvious that they could be proven immediately. Second, the conjectures were accessible to the interviewers (also the first and third authors), allowing them to follow the mathematicians' work and to ask meaningful follow-up questions.

In IS1, five of the mathematicians hold PhDs in mathematics, and one holds a $\mathrm{PhD}$ in mathematics education; all IS1 mathematicians are faculty in university mathematics departments. The IS1 interviews were individual, videotaped, and lasted approximately $60 \mathrm{~min}$. All of the IS1 mathematicians were given Conjectures 1 and 2, and three each did Conjectures 3 and 4, which were randomly assigned. After each task, we asked the mathematicians clarifying questions about their work. At the end of the interview they answered reflective questions about their example-related activity, both in the work they had done in the interview, and more generally in their own personal work. The mathematicians had approximately 15-20 min to explore each conjecture; although typically they were not able to complete proofs for each of the conjectures in the time 


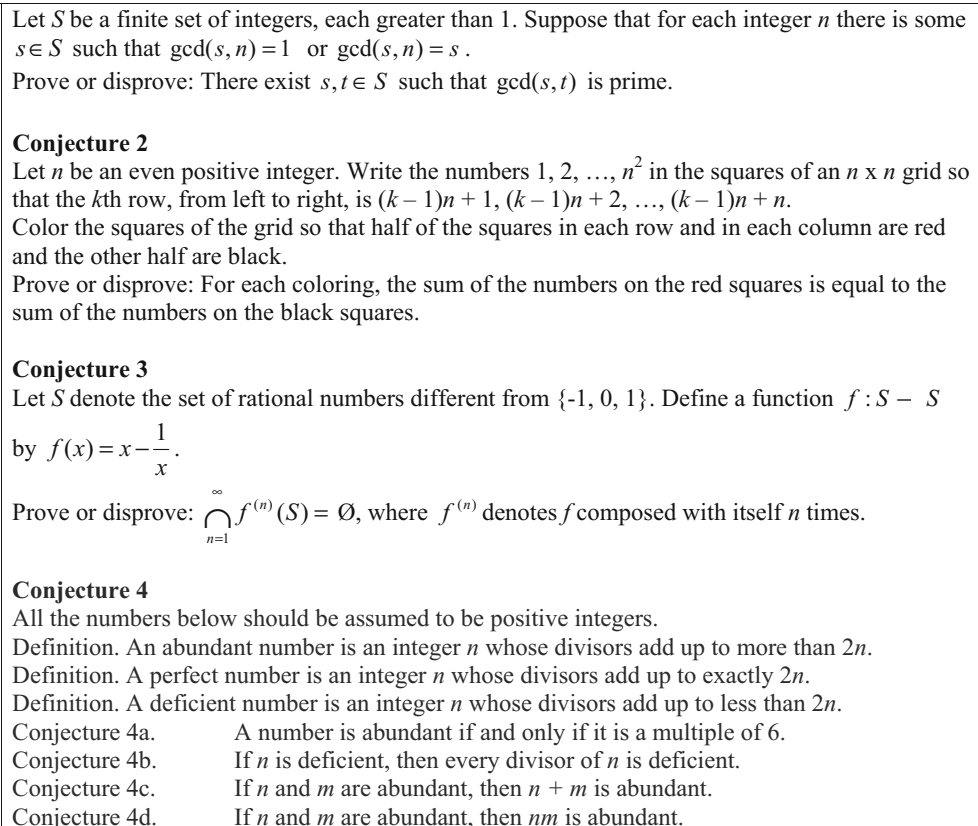

Fig. 1 Conjectures presented during IS1

allotted, they were able to make progress toward that end. These interviews were videotaped and transcribed.

In IS2, twelve of the mathematicians hold $\mathrm{PhDs}$ in mathematics, and one holds a $\mathrm{PhD}$ in computer science. All thirteen IS2 mathematicians work in a university mathematics department, six as professors, three as postdoctoral scholars, three as academic staff, and one as an emeritus professor. The IS2 interviews were individual, audiotaped via Livescribe pens, and lasted approximately $60 \mathrm{~min}$. The Livescribe pens have technology that simultaneously records what participants say and write, enabling researchers to review what is said and written in real time. All of the IS2 mathematicians worked on Task 1, and seven also worked on Task 2. During the interviews, the mathematicians were given time to work on the tasks on their own and were asked to think aloud; generally, the interviewer did not interrupt except to ask clarifying questions or to answer questions from the mathematicians. These interviews were not videotaped, but the mathematicians used Livescribe pens during the interviews, and the interviews were transcribed.

Task 1
Most positive integers can be expressed as a sum of two or more consecutive integers.
For examples, $24=7+8+9$ and $51=25+26$. A positive integer that cannot be
expressed as the sum of two or more consecutive positive integers is therefore
interesting. What are all the interesting numbers?
Task 2
Define a selfish set to be a set which has its own cardinality as an element. Find, with
proof, the number of subsets of $\{1,2, \ldots, n\}$ which are minimal selfish sets, that is,
selfish sets none of whose proper subsets is selfish.

Fig. 2 Tasks presented during IS2 


\section{Interview Data Analysis}

A member of the research team analyzed the IS1 transcripts using types, uses, and strategies that had been identified in the surveys. The process involved coding both mathematicians' observable example-related activity and their reflections about examples, and as with the surveys, the literature broadly served as a starting point for the coding. In some cases, the entire research group reviewed data excerpts that were difficult to code. These codes served to refine the initial framework, and the organizing of the codes in turn resulted in a number of themes about mathematicians' examplerelated activity in exploring and proving conjectures (Strauss and Corbin 1998). Then, two of the authors together reviewed the IS2 data, again identifying emergent themes and phenomena and reaching a consensus via discussion. We present these phenomena as the major result of this study, as they shed light on how people who are adept at proof interact with examples as they consider conjectures.

\section{Results}

In this section, we report on specific mechanisms by which examples helped mathematicians move their thinking forward as they explored mathematical conjectures, and we provide the results in two parts. First, we describe the example-related activities with which mathematicians engaged in the context of proof, which includes descriptions of ways in which mathematicians a) strategically select examples and b) strategically use examples. Second, we report on how mathematicians carry out these activities, or the mathematicians' overarching purposeful engagement with such activities. This includes ways in which mathematicians flexibly a) focus on generalization, and b) demonstrate metacognitive awareness of the role of examples in their overall proving activity. We present and discuss these findings below, drawing upon representative excerpts from both the surveys and the interviews to exemplify each theme. These findings highlight the strategic and thoughtful nature of mathematicians' example choice and use. We do not simply present a categorization of what mathematicians do. Instead, we highlight the ways in which examples serve as a site for learning, identifying how they can be leveraged in powerful and strategic ways.

Table 1 serves as an overall organization of the ways in which mathematicians selected and used examples. The categorizations both convey insight into mathematicians' example-related activity and show the rich variety of beneficial purposes for which mathematicians productively work with examples. In the subsequent sections we will explicate each category, providing excerpts from interviews and surveys to exemplify our points.

\section{Choosing and Using Examples: Example-Related Activities with Which Mathematicians Engaged}

Through our analysis we observed two broad kinds of example-related activity. These activities are interrelated, and there is some overlap between the activity types. However, we distinguish them because it illuminates two powerful ways in which mathematicians interact with examples in their proving activity. First, mathematicians 
Table 1 Example-related activities and purposeful engagement

\begin{tabular}{|c|c|c|}
\hline \multirow[t]{6}{*}{ Results Part 1} & Example-related Activities & \\
\hline & Strategic Example Choice & Strategic Example Usage \\
\hline & $\begin{array}{l}\text { Select sets of examples that increase } \\
\text { in generality and/or complexity }\end{array}$ & $\begin{array}{l}\text { Use examples to check a conjecture or } \\
\text { ascertain whether a conjecture is true } \\
\text { or false }\end{array}$ \\
\hline & $\begin{array}{l}\text { Select examples that serve as extremal } \\
\text { (or boundary) cases }\end{array}$ & $\begin{array}{l}\text { Use examples to better understand the } \\
\text { statement of the conjecture (or the } \\
\text { conjecture itself) }\end{array}$ \\
\hline & $\begin{array}{l}\text { Select examples based on the mathematical } \\
\text { properties of those examples }\end{array}$ & $\begin{array}{l}\text { Use examples to gain insight into the } \\
\text { proof of the conjecture }\end{array}$ \\
\hline & $\begin{array}{l}\text { Select examples based on their familiarity } \\
\text { with the domain at hand }\end{array}$ & $\begin{array}{l}\text { Use examples to search for potential } \\
\text { counterexamples to break the } \\
\text { conjecture }\end{array}$ \\
\hline \multirow[t]{2}{*}{ Results Part 2} & $\begin{array}{l}\text { Purposeful Engagement in Example- } \\
\text { related Activities }\end{array}$ & \\
\hline & $\begin{array}{l}\text { Focus on generalization in single examples } \\
\text { and sets of examples }\end{array}$ & $\begin{array}{l}\text { Demonstrate metacognitive awareness } \\
\text { of their activity }\end{array}$ \\
\hline
\end{tabular}

strategically selected examples as they explored conjectures and developed proofs. Second, mathematicians strategically used examples for a number of different purposes. Brief descriptions of categories for each of these activities are presented in Part 1 of Table 1.

\section{Strategic Example Choice}

In their strategic choice of examples, mathematicians intentionally put thought into which examples they would consider during their exploration of a mathematical conjecture. This stands in contrast to less sophisticated ways of selecting examples sometimes observed among secondary students (e.g., Ellis et al. 2012), in which students simply select an example with little thought or because it was the first one to came to mind. Below we discuss four ways in which the mathematicians strategically selected examples.

\section{Mathematicians Select Sets of Examples that Increase in Complexity and/or}

Generality We observed that the mathematicians described selecting examples that would be increasingly complex or increasingly general. In the interviews, one instance of this is seen in Dr. Aldridge's work described in "Interview Example 1", as he builds up from a very basic case, progresses to more complex cases, and ultimately to a more general case.

Another instance of this is seen in Dr. Chu's work on Task 1 in IS2 (for a description of the solution see "Interview Example 2"). After working on this task for some time, Dr. Chu developed a conjecture that the interesting numbers are the powers of 2. To prove his conjecture, he attempted to prove the contrapositive statement (non-powers of 2 are non-interesting) by showing that every integer with an odd factor greater than 1 can be written as the sum of 2 or more consecutive positive integers. He got stuck on the case where a number with an odd factor was divisible by 4 but not by 8 . His initial 
idea was that these numbers could always be written as the sum of 8 consecutive positive integers, which he checked with some examples.

Dr. Chu: Let's try 4 times 5; that's 20. Maybe that would be easier. Uh... so again, if I suspect that it can be written - No, it wouldn't work. It would be too small. But on the other hand, if I try something larger, like 4 times 9 , that's 36. Um, well that's a multiple of 3, so I know one way to write it as a sum of three consecutive integers. But what if I try to write it as a sum of eight consecutive integers as well? So 36 divided by 8 is 4.5 . So that would work, right? So that would be 1 plus 2 plus 3 plus 4 plus 5 plus 6 plus 7 plus 8 . Yeah, but [20 and 28] are too small for this technique. Uh... Hmm, this is interesting... And see, beyond it, uh, so if I have a number that is divisible by 4 by not by 8 and it's bigger than this, then I can use this.

Dr. Chu started with an example he considered to be easier $(4 * 5)$ and found that his method did not work because it was too small. He then considered a more complex example $(4 * 9)$ and found that his method worked. The more complex example enabled Dr. Chu to see that his method would work for $4 * k$ whenever $k$ is any odd number greater than or equal to 9 , and this is an instance of a progression toward "large," or more complex, examples.

An example of a survey response that demonstrates this activity is: "Easy ones! Start with toy cases and slowly build up the complexity. Hopefully it's obvious why what you're looking for is true in the easiest case. You can then see if that reason generalizes." There seem to be a couple of reasons why mathematicians begin with easy cases, including developing an understanding of the statement of the conjecture ("I use simple examples first, so I understand what the conjecture says and then build up to more complicated ones") or to determine quickly whether a conjecture is obviously false, ("Start with easy cases to save time in case it's false. Go from special to general, and from smaller to bigger examples (i.e. more dimensions, more nodes, more elements, or whatever the appropriate measure of complexity is"). These responses suggest that the mathematicians are aware of what even simple initial examples can do for them, and that they may gain insights into the conjecture by building toward more complex or generic examples.

In another survey response, a mathematician remarked:

I would typically start with whatever I felt the most quintessential or simple example of the type of object under consideration was - for example small integers, simply-connected topological spaces, finite abelian groups, etc. If these felt too trivial to get a sense of what is going on and why something might or might not have to be true, I'd move to the simplest interesting example - twodigit composite numbers, a torus, the symmetric group, whatever. Basically move from simplest to more complicated gradually until I got some insight into what might be important in making the conjecture true or false.

In this response, we see that (in the italicized portion in particular) the move from simple to more complicated and interesting examples is a strategic one. Here the mathematician suggests that the decision to select a progression of examples helps 
determine not only whether, but also why the conjecture might be true or false; indeed, the progression of examples can potentially yield some insight about the conjecture itself. We thus see in these responses that mathematicians sometimes select a strategically chosen progression of examples in their work with mathematical conjectures.

Mathematicians Select Examples that Serve as Extreme (or Boundary) Cases The mathematicians also noted that sometimes the search for a set of examples is connected to seeking a counterexample or breaking a conjecture. To this end, they sometimes chose extreme examples that pushed the limits of some property or that were at the edge between properties (some call these boundary cases). There are two ways in which mathematicians selected such examples. First, they chose examples that pushed the limits of a particular property or category. In the survey response below, the mathematician notes that he is considering extreme cases, "The examples I try often tend to be of extremal nature (sort of a worst or best case scenario)." Another survey response suggests that the mathematician intentionally tries to test particular values or parameters that might provide insight into the conjecture:

I try to test extremes. For example, if I conjecture that throwing a ball off a building of height 5 meters follows the equation $\exp (-t)+4$ for $t$ being time, then I would look at $t$ going to infinity and my intuition tells me the object needs to hit the ground at some point but, clearly the formula tends to 4 and not 0 so something is wrong. If the conjecture passes this test then it's worthwhile to explore further.

In the interviews, there were examples of mathematicians intentionally selecting and leveraging boundary cases in exploring and proving conjectures. Dr. Aldridge's work on the deficient number conjecture (described in detail in "Interview Example 1") is an illustration of this phenomenon from the interviews.

Second, the mathematicians chose extreme cases as potential counterexamples. In IS1, Dr. Barton provides a nice reflection on this search for counterexamples and how that can be an important aspect of working toward a proof of a conjecture.

Dr. Barton: You're trying to prove something and you go ahead and you try to prove it. And you realize that you're stuck at some point...Here's this gap. I start saying let's try, out of that gap, to build a counterexample... Then you spend some time trying to build that object. And if you can't, then you try to sort of distill why can't you? And do the reasons why you can't build that, does that now fill in the gap in your proof? If it does, great. You've now pushed your proof further or maybe you've completed the proof entirely. And if it doesn't, then it refines what...the counter-example would have to look like...And so it's this sort of back and forth trying to use that. You know, build a counterexample, and the failure or success of that to go back and look at what that says about your proof. And that dynamic back and forth can sometimes bear some fruit.

For Dr. Barton, this search for a counterexample seems to be a significant and common aspect of his proving practice. 
The survey responses also reflect that mathematicians view the search for a counterexample as valuable. For example, the mathematician with the following response notes that he first looks at simple cases to better understand the conjecture, and then he considers potential counterexamples:

First test for the most simple cases, also to understand the conjecture a little better. Then once I'm more comfortable with it, try it with some example I regard as less likely to verify the conjecture and keep looking for a counterexample. Eventually, if I figure out the conjecture is true for all the examples tested, the search for a counterexample should have given me some insight in how to prove it.

Another survey responder said:

I try to "break" the conjecture; that is, I choose examples that are likely to show the conjecture is not true. For number-based conjectures, I choose 0, numbers close to 0 (both positive and negative), very large and very small numbers, for examples, both integers and non-integers.

This response highlights the fact that the selection of extreme cases can be closely related to a use of breaking a conjecture, but here we highlight the kind of example being chosen. This response reveals a mathematician considering potential examples that are extremes in some way - unusual numbers, or very large or small numbers. Searching for and selecting such examples can play an important role in shedding light on how to prove the conjecture at hand. We follow up with this finding in the section "Mathematicians Use Examples to Flexibly Leverage a Dynamic, Back and Forth Relationship Between Examples (and Proof) and Counterexamples (and Disproof)", which further addresses mathematicians' attention to the back and forth relationship between proving and a search for counterexamples.

\section{Mathematicians Select Examples Based on the Mathematical Properties of Those}

Examples The mathematicians were keenly aware of the mathematical properties of their examples, and they capitalized on their understanding of such properties in order to select certain types of desired examples. This supports related findings by Weber (2008), who reports that some mathematicians drew upon mathematical properties as they validated proofs. In the interviews, the mathematicians' domain-specific expertise was apparent as they spoke about the mathematical features of their examples, such as choosing a number with many factors or creating a set with no primes. This emphasis on properties appeared most frequently with Conjecture 1 in IS1, as the mathematicians drew upon their knowledge of mathematical concepts such as primeness, common divisors, and the fundamental theorem of arithmetic to consider examples or counterexamples. As an example, Dr. Leonard constructed a set $\{4,8,12,20\}$ in an attempt to derive a counterexample. He had recognized that a counterexample must not have primes in it, and the excerpt below highlights his consideration of specific mathematical properties as he attempted to construct a possible counterexample and proceed with the problem. Dr. Leonard displays knowledge of elementary number theory as he carefully selects four numbers that are not prime and that all have a 
composite number as a greatest common divisor. Facility with specific mathematical properties enabled him to make sophisticated decisions in constructing an example.

Dr. Leonard: The greatest common divisor between the two of them [looking at the statement of the conclusion] is not prime... Okay, it would have to be some set like 4 [writes $\{4,8,12,20\}]$. That would be...their greatest common divisor is not prime.

We observed this same phenomenon in the surveys as well. In the following survey response, the mathematician was attuned to properties such as sign, rationality, identities, and number of factors in trying to test the conjecture:

I'll test different types of numbers - is it true for both positive and negative integers? Is it true for rational and irrational numbers? Complex numbers? Does it work for the identity (different depending on the problem at hand)? I might also look at extremes (If I'm testing an idea with 4-digit numbers, I'll test high and low extremes). I tend to try with "nice" numbers first to make my life easier. I work in number theory so I'll test manageable numbers with many factors ( 12 is a favourite).

In this response, specific mathematical features affect example choice and generation, and the mathematician seems aware that selecting different examples with different properties can reap respective benefits. For instance, by trying "nice" numbers, which might satisfy some set of properties, she might gain easier access to the conjecture. Here she specifies that choosing a dissimilar set of numbers to test might involve numbers with widely different properties. In sum, we see mathematicians drawing upon their knowledge of various mathematical properties as they choose examples. This phenomenon demonstrates that they are conscious about the mathematical properties that might make an example interesting or worthwhile to consider in a particular context.

\section{Mathematicians Select Examples Based on Their Familiarity with the Domain at}

Hand Mathematicians' extensive domain knowledge also appears to play a critical role in how they chose examples. The notion of domain knowledge affecting mathematical practice is brought up by a number of researchers. For example, in his discussion of problem solving resources, Schoenfeld (1985) notes "the successful implementation of heuristic strategies in any particular domain often depends heavily on the possession of specific subject matter knowledge" (p. 92). Within proof, Weber (2008) found that "some participants' validation standards and strategies were dependent on their familiarity with the domain that they were investigating" (p. 447). Regarding examples, Rittle-Johnson et al. (2009) indicate that prior knowledge was an important factor for middle school students as they compared examples, and a number of other researchers (e.g., Sandefur, et al. 2013; Stylianides and Stylianides 2009) argue for the importance of familiarity and domain in examples via Watson and Mason's (2005) notion of students' personal example space. In terms of domain knowledge, the mathematicians noted that context and familiarity have a direct effect on their selection of examples, often enabling them to make well-informed choices. Some of the interview 
participants indicated that if they were working in a domain they knew well, they would regularly draw upon familiar, or "stock," examples. For instance, for Conjecture 4a, Dr. Hickson used his familiarity with the fact that 6 is a perfect number to make progress on that task, as seen in the exchange below.

Dr. Hickson: Conjecture 4a: A number is abundant if and only if it is a multiple of six. Hmm, okay. So an example immediately comes to mind. Six is a perfect number, and so that's going to be false if you are allowed to take a trivial multiple of six. So $[\ldots]$

Interviewer: Okay. And that you knew six was a perfect number from experience.

Dr. Hickson: Yeah, that one I just happen to know.

The following survey response suggests a reason for selecting such familiar examples. One mathematician noted, "First, I try something I know a lot about - a space or function that I have worked with a lot and know lots of properties of. Next, I try something slightly more obscure, and eventually I get into a general case." This response indicates that by selecting a familiar space or function, the mathematician has at her disposal rich information about certain features and properties of that example. Presumably, such information can contribute to more meaningful work with that example in the context of the conjecture.

The mathematicians were also aware that the particular mathematical context of the conjecture could determine whether examples might be accessible or useful. For instance, the following response suggests awareness that context matters, "It depends on the subject as well. In geometry I think examples are useful (somehow it is relatively easy to see what the 'generic case' is). Whereas in graph theory I find that looking at simple examples is typically misleading." Mathematicians also indicated that in some cases examples were very difficult to construct, and that sometimes it was not trivial (or even possible) to construct an example in their field of study. One mathematician stated, "In my work...the invariants I work with are incredibly hard to compute...," and another noted that, "In my mathematical experience, the trick is to FIND examples." It is noteworthy that this difficulty may set the work of expert mathematicians apart from the work of other populations. In middle or high school mathematics, for instance, the intrinsic nature of the mathematics involved does not pose as much of a challenge to finding examples.

\section{Strategic Example Use}

We also found that mathematicians used examples for a variety of different reasons, and these uses reflected intentional courses of action that were aimed at contributing to their exploration and proof of the conjecture. That is, the mathematicians demonstrated that they thought carefully about different ways to leverage examples in their work. Again this kind of activity reveals more intentionality than is commonly seen among students, who tend to use examples in more limited capacities in their proof-related activity. Below we discuss four different ways in which the participants used examples. 


\begin{abstract}
Mathematicians Use Examples to Check a Conjecture, Both for Reasonability and to Ascertain Whether or not a Conjecture Might be True We first saw that mathematicians would use examples simply to check the conjecture, often to see if the conjecture was even reasonable. For instance, in IS1 Dr. Hickson explored a couple of small examples while working on Conjecture 3. The following exchange (particularly the italicized section) demonstrates this tendency to check. Dr. Hickson selected a simple example with the intent of using that example to check what it might mean for an example to satisfy the conjecture.
\end{abstract}

Interviewer: I think you mentioned at one point, I think you called your first example a toy example?

Dr. Hickson: Mm-hmm. The one with the two?

Interviewer: Well what do you mean by that?

Dr. Hickson: Ah, well just in the sense that I don't think there's any reason to expect that that's the only kind of example out there. But it's something I can run through the wording, and see whether or not it [the example] satisfies it [the conjecture].

Many survey responses also indicated that examples were commonly used to check the conjecture. For example, the following survey response suggests that a first step when exploring a conjecture is simply to check that conjecture with an easy example. Then, if it works, she could continue with less trivial examples: "I would start with an example that is easy to check. If that example works out and agrees with the conjecture I would check a less trivial example then." Another mathematician indicated that examples can serve the purpose of ensuring that the conjecture is at least reasonable: "Simple examples that are easy to test and expected to work out, to check that the conjecture is not completely wrong." These responses highlight the strong degree of intersection between example choice and example use.

The mathematicians also used examples to decide whether they should try to prove or disprove a conjecture. Harel and Sowder (1998) refer to this as ascertaining. Below, in his work on Conjecture 2, Dr. Wells concludes that coming up with a particular example that satisfied the conjecture suggested that he should attempt to prove (as opposed to disprove) the conjecture.

Dr. Wells: Well, first I was trying to make sense of the hypothesis, to make sure the hypothesis was even possible...And then once I convinced myself that the hypothesis was possible, in the example of the set being two... Then I was able to use that example to show that in the case of that example, the conjecture worked. And so that convinced me to try to prove the conjecture rather than to disprove it.

In the following survey response, the mathematician indicates that in instances when he is unsure of whether or not the conjecture is true, he tries to find simple examples to work with computationally. 
Any conjecture that is not obviously true is hard. Hence I try to come up with situations which are simple enough that I can make explicit calculations (eg. most facts about complex geometry, I will test on the Riemann sphere/ $\mathrm{CP}^{\wedge} 1$, or a complex torus, since doing explicit calculations on other manifolds is very difficult). If these examples fail to illuminate anything new (because they are too low dimensional for example), I try the conjecture on a 4 dimensional ball, or $\mathrm{CP}^{\wedge} 2$, then $\mathrm{CP}^{\wedge} 3$, and then $\mathrm{CP}^{\wedge} \mathrm{n}$; then $\mathrm{I}$ try it on bigger spaces like Grassmannians. If it works in these examples, then I would guess it is true.

The last line of this response shows that he uses examples to gain insight into whether the conjecture might in fact be true. Thus we see that mathematicians use examples to check the reasonability of conjectures and to help them ascertain whether a conjecture might be true or false.

Mathematicians Use Examples to Better Understand the Conjecture The mathematicians also use examples in an effort to better understand a conjecture. Often this means that they will explore a concrete example as a means to develop an understanding of what a conjecture is stating. As an example of this, in IS1 Dr. Hickson was working on Conjecture 2. He spent time considering some small examples, and the interviewer questioned him about his work. His response highlights the fact that he explored small examples in order to better understand what the conjecture was saying, especially because the conjecture was difficult for him to parse initially.

Dr. Hickson: This is a hard assumption to, for me, anyway, to process. Like there's some quantifiers in there. And there's a weird kind of combination of there exists something relatively prime to it, so I had to restate it a few times. I wanted just to check it on some numbers just to see it play out.

In the surveys, we see the mathematicians using examples to "understand the conjecture a little better," as one response put it. The following survey response highlights this use of examples in more detail. He describes how examples can be used as a way to confirm that one understands the statement of the conjecture. Even more, the response suggests that the examples (and even the search for examples) can serve to clarify the conjecture and its hypotheses:

I first try to choose trivial examples to make sure I understand the statement of the conjecture and to possibly identify aspects of the conjecture that are non-trivial (sometimes even trying to identify such examples helps me in this). Then I try to find the simplest non-trivial example I can, and I try to understand these in light of the conjecture. If I don't know much about the history of the conjecture it is often difficult for me to find explicit examples, even simple ones. If this is the case I just try to find examples that satisfy some of the hypotheses, but possibly not others, and then try to understand why such examples are not included in the statement of the conjecture.

Mathematicians Use Examples to Gain Insight into How a Proof Might Develop Many of the mathematicians discussed using examples in order to make 
significant steps toward a proof. In Task 1 in IS2, Dr. Chu conjectured that the interesting numbers were exactly the powers of 2 (he is correct; see "Interview Example 2" for a solution). He was attempting to prove one direction of his conjecture, that if a number is not interesting, then it is not a power of 2 , when he had the following insight:

Dr. Chu: Hmm... Well clearly, if it can be written as a sum of an odd number of consecutive integers, then it cannot be a power of 2 .

Interviewer: Why is that?

Dr. Chu: Well, 'cause - take this one [points to 20]. Right? That's...2 plus 3 plus 4 plus 5 plus 6 . And it's a sum of an odd number... of consecutive integers. Right? So that means that you can write it as the middle number times 5 .

Dr. Chu went on to make a similar argument for the sum of an even number of numbers, using 36 as his example.

Dr. Chu: Now if it can be written as the sum of an even number of consecutive integers... ah, yes. So for example, the 36 . Oh, it can be written as the sum of eight consecutive integers. Right? If you find the mean, then you will get 4.5.

Interviewer: Right.

Dr. Chu: Now, you have eight of them, so you can take the 2 from 8 , and you would multiply that to 4.5 , and that would give you an odd factor.

The numbers 20 and 36 served as generic examples for Dr. Chu, allowing him to explain why the sum of any number of consecutive numbers has an odd factor. He chose 20 and 36 because they had the properties of being the sums of an odd and even number of consecutive numbers, respectively, and he used these examples to formulate an argument about what kinds of numbers might be interesting. In his explanations, he called upon the property of sets of numbers that the sum of a set of numbers is equal to the mean of that set times the number of numbers. He then connected his explanations back to his conjecture using the fact that powers of 2 have no odd factors other than 1. Altogether, Dr. Chu's use of examples led to a proof of one part of his conjecture, demonstrating the role of examples as a key part of his proof development.

The surveys revealed a number of responses in which mathematicians reflected that they would choose and select examples in order to gain insight into how they might formulate a proof for the conjecture. For example, one mathematician said:

I typically begin with what I believe to be the simplest example. I then try to prove the conjecture for this example. If I succeed, I then try proving it for harder examples. If I'm able to prove a statement directly for every example I can think of, I can usually then see how to write a general proof. If I fail in proving the 
conjecture but am unable to show it is false, I analyze why it is I'm having trouble proving the conjecture and try to find a different example where I might not run into this difficulty (emphasis added).

The italicized portion in particular highlights that mathematicians are aware that examining their examples can lead to insights into how to construct a more general proof.

\section{Mathematicians Use Examples to Flexibly Leverage a Dynamic, Back and Forth Relationship Between Examples (and Proof) and Counterexamples (and}

Disproof) The mathematicians also reported a complementary relationship between proving and disproving, which is intrinsically connected to a search for counterexamples. Here we see that a search for counterexamples and a search for a proof are closely related, and mathematicians tend to leverage that relationship in their proof-related activity.

In the interviews, a number of the mathematicians discussed the role of counterexamples in their proving process, noting that as they attempt to develop a proof, they engage in a back and forth process of formulating a proof and considering counterexamples. They described starting out by attempting to prove a conjecture, but then may get stuck, stop, and search for a counterexample. This search for (or inability to find) a counterexample might then provide insight into the development of the proof. As an example of this, we recall Dr. Barton's reflection about his work with examples (in IS1). He had previously articulated this relationship in his discussion of counterexamples, and he says, "And so it's this sort of back and forth trying to use that. You know build a counter example and the failure or success of that to go back and look at what that says about your proof. And that dynamic back and forth can sometimes bear some fruit."

In reflecting on the role of examples at the end of his interview, Dr. Hickson said the following, which highlights the back and forth process that a mathematician may go through:

Dr. Hickson: You know the height of folly is to assume you know the answer when you don't. So you should always give some effort to both sides of the problem. And so I try to go back and forth. I'm like any other person though. I'm going to avoid work when I can. I'm going to pursue the easier option first... if I run out of gas then I'm going to reluctantly try the other one.

In the survey data, several mathematicians indicated that maneuvering this back and forth process was an integral part of their example-related activity. One mathematician responded to the prompt by saying:

First test the easiest cases. (E.g., for integers, test 2, 3, 4, 5, 10) Then test something that is qualitatively different from the easiest cases. If it still works, make a first attempt at a proof. If you can’t prove it, try to cook up counterexamples that exploit the holes in your "proof." If you can't make counterexamples, use what you learned from the failed counter-examples to fix the holes in the proof. Go back and forth between proof and disproof, using the failures of each side of the argument to build up your attempt on the other side.

This response highlights the mathematician's awareness of how counterexamples in particular can serve to provide insight into what a proof might involve. He indicates that 
trying to construct counterexamples can be an enlightening process, and that even a failed search for counterexamples can bolster a proof attempt. Another survey response highlights the "hand in hand" nature of the simultaneous proof for a proof and counterexamples:

First step usually is to think of simple examples in case there is an easy counterexample. After that the search for a proof of the conjecture and counterexamples typically go hand in hand. That is, a proof often depends on a series of lemmas, and each of those lemmas reduces the complexity of the conjecture and typically makes finding a proof or counterexample easier. Of course the failure of a lemma does not usually eliminate the conjecture, but it at least eliminates one path to a possible proof. Often in parallel one also thinks of corollaries to the conjecture, since that also can help find counterexamples.

This mathematician's response focuses on counterexamples, and he suggests that counterexamples can be effective ways of eliminating potential paths to a proof. By focusing on proving lemmas, which might be easier to disprove via counterexamples, the mathematician can try to hone in on a proof. These examples from the surveys suggest that some mathematicians are keenly aware of exactly how useful examples and counterexamples can be in the proving process, and that the proving and disproving processes are each closely linked to the generation of (or attempts to generate) examples.

\section{How Mathematicians Work with Examples - the Purposeful Engagement that Guides Mathematicians' Example-Related Activity}

There were two aspects of mathematicians' exploration and proving of conjectures that guided and directed their example-related activities of selecting and using examples. This purposeful engagement included a) focusing on generalization via leveraging structure in a particular example or set of examples, and b) displaying an overall metacognitive awareness of their proofs and how examples related to their proofs. In this section, rather than providing interview and survey excerpts for each of these types of purposeful engagement, we first briefly describe and provide survey responses for each type. We then provide two in-depth interview excerpts and discuss how the purposeful engagement is demonstrated by these lengthier episodes.

\section{Mathematicians Focus on (and Leverage) Structure Among Particular Examples or Sets of Examples by Searching for Patterns that Might Lead to Generalization}

On several occasions mathematicians used specific features of an example in order to make significant steps toward a proof. In these instances, the mathematicians grounded their thinking in a particular example, and by manipulating that example they developed an idea for how a more general proof might develop. A number of researchers have suggested that proofs can emerge from work with examples (e.g., Sandefur et al. 2013; Weber 2008), and more specifically in terms of a generic example (e.g., Pedemonte and Buchbinder 2012). One survey response demonstrates that this mathematician can move toward generalization based on observations from basic examples: "I might also look for simpler examples that contain enough structure to be generalized. Or I look at "building 
block" types of examples. If I can show a conjecture for "building blocks," it may help me show it in general. This picture would suggest patterns or sub-conjectures that I could prove." Here the mathematician specifically discussed the fact that he might be able to identify structure in a simple example that might help facilitate generalization.

Regarding the identification of structure among a set of examples, Harel (2008) discussed patterns in his presentation of DNR-based ${ }^{2}$ instruction of mathematical induction, and Weber (2008) reported a handful of instances in which mathematicians accepted a statement as valid based on a pattern that emerged from several examples. More specifically, Harel differentiated between result pattern generalization, which is "a way of thinking in which one's proving is based solely on regularity in the result" (p. 491), and process pattern generalization, which is "a way of thinking in which one's proving is based on regularity in the process" (p. 491). Depending on the situation or the nature of the conjecture, we see evidence among the mathematicians of both of these ways of thinking.

To exemplify this phenomenon of using patterning to facilitate generalization, we highlight a couple of survey responses in which mathematicians expressed attention to patterns. One noted that:

Typically, when testing conjectures I have to find a compromise point between "general enough" (say, an ideal generated by randomly generated homogeneous ideals of degree n) vs. something computationally feasible (three such generators, or all generators of degree 3, or all generators powers of linear forms, or monomial ideals, etc.).

This mathematician went on to say: "If the "general" case is too computationally infeasible, I'll enumerate every possible example of low degree/dimension to see what the numerics there look like, then attempt to find a general pattern." The latter portion of this response suggests that identifying a pattern in many simple examples can facilitate the formation of a general case that might otherwise be unattainable. Here the patternseeking activity seems to play a particular role in helping to generate a general case, and we take this to be representative of Harel's (2001) result pattern generalization, as the mathematician seems to be attuned to the actual "numerics" of the pattern. Another mathematician noted that in one part of her work with examples, she would:

Look for the simplest possible example first ( 2 by 2 matrix, for example, or group with 2 or 3 elements), gradually do more complicated ones if the simple one didn't give enough information. Look for patterns that hold for 2 or more examples, and that seem like they're susceptible to induction or similar arguments. Look for characteristics that are slightly different between two examples, and try to figure out why.

Here, the development of multiple examples is not simply meant to provide a set of examples, but there is an intentional aim of selecting examples with the development of a pattern in mind. The mathematician seems aware that the patterns that hold for at least two examples might give her some idea about what a more general argument might entail, suggesting process pattern generalization.

\footnotetext{
${ }^{2}$ Harel (2008) proposed instruction based on three principles - duality (D), necessity (N), and repeated reasoning $(\mathrm{R})$.
} 


\section{Mathematicians Demonstrated an Overall Metacognitive Awareness of Their Work with Examples and how that Related to Their Broader Proof Activity}

In discussing and working with examples, mathematicians overwhelmingly exhibited a meta-awareness of their activity. They were able to situate their example-related activity in terms of a broader context of their mathematical work and they demonstrated intentionality about their work with examples. They were aware of what their examples could do for them and were often explicitly deliberate about their example choices. Furthermore, mathematicians are aware of their overall proof activity and are intentional about their example-related activity and how examples play into this activity. Such metacognition has been reported as being an important aspect of mathematical activity (e.g., Schoenfeld 1979, 1992), and particularly among mathematicians' practices such as proving (Savic 2012) and problem solving (Carlson and Bloom 2005).

The following survey response exemplifies this tendency, as the mathematician displays a clear strategy and cognizance when it comes to choosing examples when exploring a conjecture:

If possible, I try and figure out how a proof might go. When I run into a "difficult" part of the idea, I then begin trying to craft an example that exposes this difficulty. If this difficulty can never be avoided, then said example may be a counterexample. If said example is not a counterexample, then I try and prove the conjecture. If I still fail, then I may look at my conditions and try and weaken them so as to bypass the troublesome part more easily. I then repeat the above process.

This mathematician seems to be aware of how exactly he uses examples when working on a conjecture. Here, it seems that an example can provide insight to a difficult step in a proof, refute the conjecture (if it turns out to be a counterexample), or lead to a revised conjecture.

Additionally, some mathematicians' survey responses indicate that they recognize that their strategy might change depending on whether they think the statement to be true or false. One such responses was:

If I am not sure whether the conjecture is true, I start by considering an example for which I believe it will be true- maybe a special case of the hypotheses of the conjecture, and then if it succeeds, I would try to consider a more general example. If I think it is not true, I choose the simplest example for which I believe the conjecture will fail. If I am fairly certain it is true, I usually try to consider the most general case- i.e. draw a picture that breaks any extra assumptions that I think might obscure the story.

According to this response, this mathematician seems aware of the ways in which examples can shed light on the truth or falsity of a conjecture. She describes strategic guiding principles for selecting examples in trying to determine the validity of a conjecture. This suggests that mathematicians are aware of how their choices and uses of examples can function in different ways as they engage in a variety of proof-related activities. 
To summarize the themes so far, we have highlighted several key features of the mathematicians' example-related activity. We demonstrated the varied and robust ways in which mathematicians strategically select and use examples, and we also highlighted the purposeful engagement with examples that guides their activity, especially through attention to generalization and acute metacognitive awareness. In order to exemplify and explore the interplay between these themes in mathematicians' work, we now present a detailed discussion of two longer interview excerpts.

\section{Interview Examples}

By providing significant mathematical detail in the following two in-depth interview excerpts, we unpack and demonstrate the productive ways in which examples actually arise during mathematicians' exploration and proving of conjectures. These examples also serve to show that many of the activities and purposeful engagement with examples occur simultaneously and are richly connected across the mathematicians' work.

Interview Example 1 As a rich example of the ways in which mathematicians can powerfully and strategically leverage example-related activity in the proving process, we highlight how Dr. Aldridge's work with a particular example allowed him to make general arguments and ultimately formulate a valid proof of Conjecture $4 \mathrm{~b}$, which states: If $n$ is deficient, then every divisor of $n$ is deficient).

Dr. Aldridge proved this conjecture by proving the contrapositive of the statement (that a number with at least one non-deficient factor must itself be non-deficient). We give a brief summary of his proof here to provide the mathematical idea of the proof, and we then present the role examples played in formulating this proof (see Fig. 3). Dr. Aldridge argued that if a number $b$ has a factor $a$ that is not deficient, then $a$ has proper factors $f_{l}$ through $f_{k}$ whose sum is greater than or equal to $a$. Since $a$ is a factor of $b$, there is a number $d$ such that $b=a^{*} d$. Then $d^{*} f_{l}$ through $d^{*} f_{k}$ must also be factors of $b$ (and since $f_{l}$ through $f_{k}$ are strictly less than $a, d^{*} f_{l}$ through $d^{*} f_{k}$ must be strictly less than $b$ ). He noted that the sum of $d^{*} f_{1}$ through $d^{*} f_{k}$ must be greater than or equal to $d^{*} a$, which is itself already a copy of $b$. The sum of $b$ 's factors, then, includes $d^{*} f_{1}$ through $d^{*} f_{k}$, which is greater or equal to $d^{*} a$, and $b$ itself. This is greater than or equal to two copies of $b$, and thus by the definition of deficient, $b$ itself cannot be deficient.

In his initial work on this conjecture, Dr. Aldridge examined what he called "test cases," in which he drew upon the perfectness of 6 to examine numbers in which 6 was a factor. His rationale for this is seen below.

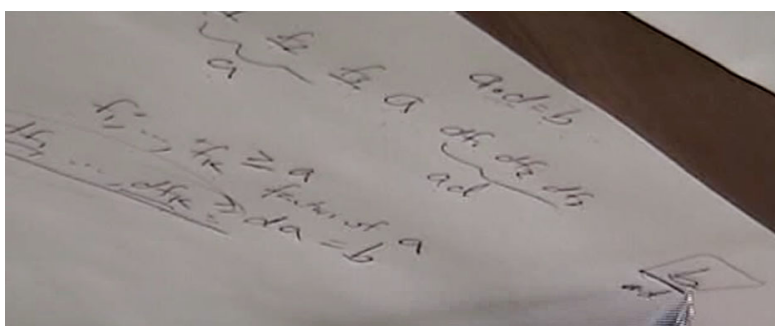

Fig. 3 Dr. Aldridge's proof of conjecture $4 b$ 
Dr. Aldridge: And then the real reason why I went after it with examples, not so much that I thought these would be counterexamples, as I thought they would be good test cases. And they'd maybe give me a feel for how, more information as to maybe why this is true.

Interviewer: Okay, and what do you mean by test case?

Dr. Aldridge: Um, test case because the 6, like I said before is perfect. So it's going to be, it's a, it's a pretty decent, uh, example of maybe, it's, so if anything has a chance to be a divisor that's not deficient inside of number that is deficient... I would guess it would be a perfect number [he previously shared that he was aware of the property of perfectness].

Continuing to focus on 6 , after trying to see if $6 * 2$ and $6 * 3$ would have to be abundant, he chose an example of $6 * 11$. While working through this example, he had the following insight (illustrated in Fig. 4).

Dr. Aldridge: It's almost like you get, like a duplication of the perfectness of six that shows up in this piece here.

Interviewer: Okay, how so?

Dr. Aldridge: So, so, like this one, two, three adds up to six. Eleven, twenty-two, thirty-three actually adds up to sixty-six. So I'm feeling like I probably ought to be able to prove that this is a true statement.

This exchange provides evidence that Dr. Aldridge used examples to ground his work in something concrete, particularly when he needed to make sense of a given situation or conjecture. Dr. Aldridge's activity puts a slight twist on the manipulation stage of the manipulating-getting-a-sense-of-articulating (e.g., Sandefur et al. 2013), because while he talked about manipulating a concrete example to get a sense of the conjecture, he did so in a domain with which he was less familiar (although the example he chose may have been familiar to him).

His work with this example not only confirmed that he thought he could prove the conjecture, but using $6 * 11$ as a generic example ultimately led him to a sketch of the correct proof described above. As he wrote out a sketch of the proof, he simultaneously referred back to his example in doing so (Fig. 5 shows him referring back to the particular example in his write-up of the proof - in his notation, $b$ is the number chosen

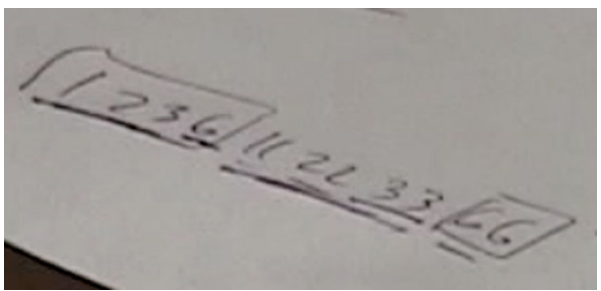

Fig. 4 Dr. Aldridge pointing out the "duplication of the perfectness of 6." 


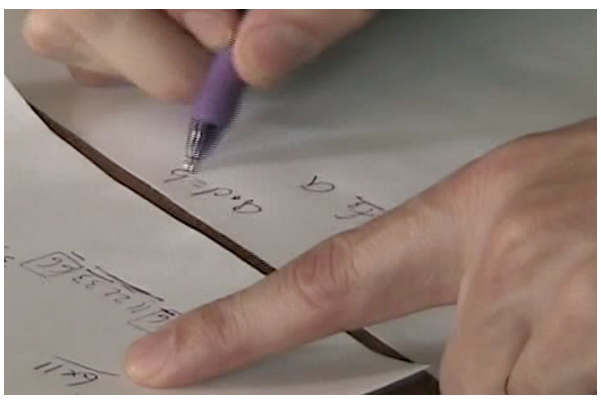

Fig. 5 Dr. Aldridge refers to the particular example in writing up the proof

as the example $(b=66), a$ is the factor of interest $(a=6)$, and $d$ is the number that satisfies $\left.b=a^{*} d(d=11)\right)$. The connection between the $6^{*} 11$ and the $a, d$, and $b$ enabled him to formulate the proof, as described above (see Fig. 3).

What is most interesting to us is not that he proved the conjecture, but rather the precise role that his example $6^{*} 11=66$ played in his development of this proof. In reflecting on his proof, Dr. Aldridge made several statements that highlighted the importance of the example. Specifically, the nature of the multiplication by 11 allowed him to see that certain numbers (11 times the factors of 6 ) would show up in the complete list of factors. While this property (that $d$ times the factors of $a$ will appear in the list of factors of $b=a^{*} d$ ) is a property that he asserted, "is clearly always going to work out," he acknowledged that the nature of the number 11 made that particularly salient for him.

The structure of the example enabled him to recognize a key piece of the proof. This focus on structure is seen in the following excerpt, in which the interviewer asked him to reflect on the role of the $6 * 11$ example in his proof.

Interviewer: You said something about, maybe, start, being able to start a proof based on that observation you found in that six times eleven...

Dr. Aldridge: Right, which is kind of what I was thinking of was that the perfectness of the, um, 6 is basically copied, replicated by these multiples. Each multiple of the factor of 6 . So I've got my 1, 2, 3, 6 here. Right now the 1, 2, 3, adds up to 6 . And then multiply each of those by 11 , those are also in my list of factors, and I add those up, I get 66 . Which, kind of is clearly always going work out... But then when I wrote it down it was actually quite helpful because, I mean, I was also benefited by the choice of $11 \ldots$ Because you multiply by 11 , it looks very much like the number you started with before you multiplied by $11 \ldots$ And so, it was much more transparent that the structure on this 11, 22, 33, 66 mirrored the 1, 2, 3, 6 .

In this interview episode, we see Dr. Aldridge strategically selecting and using examples, and we see both the generalization piece and the metacognitive piece at play. He chose a simple boundary case, and from there he selected a set of examples that progressed according to certain mathematical properties. Then, he was able to focus on one particular example (the $6 * 11$ ) and use its structure to build an argument for the general case. This highlights the role that generalization played in how he leveraged his choice of examples into a productive and correct proof. In addition, throughout his work on this problem, it is clear that he was aware of his overall process, 
both what he was trying to do at each stage and why he was doing it. He demonstrated strategic intentionality as he worked, looking ahead and looking back, which highlights the kind of metacognitive awareness we previously described.

Interview Example 2 As a second example from the interviews, we consider Dr. Weisman's work on Task 1 in IS2, for which he was ultimately able to provide a correct conjecture and proof. To provide some context for Dr. Weisman's work, we give a sketch of a solution to Task 1 (see Fig. 1 for the statement of Task 1). It can be shown that the sum of two or more consecutive positive integers has an odd factor greater than 1. Specifically, the sum of $2 k+1$ consecutive integers starting at $n$ has an odd factor of $2 k+1$ and the sum of $2 k$ consecutive integers starting at $n$ has an odd factor of $2 n+$ $2 k$ - 1 . Conversely, if a positive integer $N$ has an odd factor $m>1$, it can be shown that $N$ can be written as the sum of either $m$ or $2 \mathrm{~N} / \mathrm{m}$ consecutive positive integers, whichever is smaller. The interesting numbers are thus exactly those positive integers that have no odd factors greater than 1. In other words, the interesting numbers are the powers of 2.

Dr. Weisman had been given the task just prior to the exchange below. Before beginning to work on the task, he described three possible strategies.

Dr. Weisman: Okay...So there's a kind of gradation here. We could look for numbers that are, um, sort of level 2 interesting, which would mean they're not expressible as the sum of just two positive integers. We can look at level 3 interesting, and so forth. And...maybe those sub problems might be easier to understand. And once we understand those sub problems, then we put our results together... So that would be one approach.

[long pause], Um, let's see now. Let me just, before I start doing any calculations, let me just kind of free associate a little bit. We could list out the positive integers. Imagine them on some kind of a number line. And...so what we're doing, if we consider now, the non-interesting numbers for a minute, we're taking some interval in that list, of any length, and then we're going add up those numbers involved, and whatever we get, that's a non-interesting number...

Dr. Weisman then paused for a while in order to think. We observe that at this point, we already have evidence of his metacognition. Dr. Weisman is already strategizing ahead of time how to tackle the problem and to consider what sorts of examples he should try. He also anticipates a possible route before actually digging into the work. After some time, Dr. Weisman made the following comments, demonstrating that he was aware that he could take another approach to the problem.

Dr. Weisman: I suppose another way of...thinking about this is to, um, notice that... well let me define a new term. A very non-interesting number would be that is the sum of $1+2+3$ up to whatever, something.

Interviewer: Okay.

Dr. Weisman: Okay. Very non-interesting. So any non-interesting number is gonna be the difference between two of those, 'cause you're gonna, you start at 
$\mathrm{A}$ and you go to B. That's just like going from 1 to $\mathrm{A}, 1$ to $\mathrm{B}$, and then taking the difference between the two, or maybe 1 to $\mathrm{A}-1$, and then taking the 1 to $\mathrm{B}, 1$ to A minus 1, taking the difference would be just like going from $\mathrm{A}$ to $\mathrm{B}$.

Interviewer: Right. Sure.

Dr. Weisman: So...if we could get a good grip on...the very non-interesting numbers, okay, but those are easy. Because those are just binomial coefficients.

The first strategy Dr. Weisman described involves breaking the task into subproblems, because, "maybe those sub-problems might be easier to understand." By his definition, the interesting numbers are the numbers that are level $n$ interesting for every number $n$. Thus, if he can determine which numbers are level $n$ interesting for each $n$, then he will know which numbers are interesting.

For the second and third strategies, Dr. Weisman approached the task from the opposite perspective - looking for the numbers that are not interesting. In the second strategy, he described a concrete approach to constructing examples of noninteresting numbers: "We could list out the positive integers. Imagine them on, uh, some kind of a number line...we're taking some interval in that list, of any length, and then we're going to add up those numbers involved, and whatever we get, that's a non-interesting number." In the third strategy, he suggested looking at a special class of examples of non-interesting numbers - the numbers that are sums of consecutive numbers starting with 1 . He observed that these examples, which he calls "very non-interesting," are useful to him because any non-interesting number is the difference of two very non-interesting numbers. He also observed that the very non-interesting numbers are "easy," because they are familiar to him as binomial coefficients. His familiarity with the very non-interesting numbers led him to proceed with the third strategy, through which he ultimately developed a correct conjecture and proof. Dr. Weisman articulated a number of different approaches he could take toward the problem, and he identified different classes of examples that would be relevant in the respective approaches. We highlight this example because it demonstrates sophisticated metacognitive awareness of how he was approaching the problem and how examples were arising in the problem.

Summarizing Examples 1 and 2 Both of these examples serve to exhibit the purposeful engagement with example-related activity that we observed among the mathematicians. We see in both instances that Dr. Aldridge and Dr. Weisman demonstrated intentional ways of selecting and using examples, and they were reflective about the various ways in which examples might prove useful in their work. Dr. Aldridge's work especially highlights how he purposefully selected a progression of examples, and then he leveraged structure in a particular, intentionally chosen example in order to generalize and arrive at a correct proof. Dr. Weisman leveraged different classes of examples as he clearly articulated and considered a number of different approaches toward the task. For both of these mathematicians, their overall metacognitive awareness of the variety of ways in which examples might productively arise in their work was evident. Thus, these two examples serve as cases that highlight the sophisticated ways in which mathematicians can purposefully engage with examples as they explore and prove mathematical conjectures. 


\section{Discussion and Implications}

In this paper, we have presented several findings about mathematicians' examplerelated activity when exploring and proving conjectures, which help to paint a more complete picture of the variety of ways in which mathematicians draw upon examples. Our findings have extended the existing literature, especially work initially done by Antonini (2006) and Iannone et al. (2011), which introduced categorizations of example generation. We have built on those existing frameworks by suggesting our own characterization for mathematicians' example generation (Table 1). We have demonstrated the ways in which mathematicians strategically choose and use examples, and we have emphasized the attention to generalization and the metacognition that reinforces such activity. In this section we offer a couple of points of discussion, connecting these to pedagogical implications and suggesting directions for future research.

The behaviors we see in mathematicians' activity are not entirely dissimilar from what has been reported among students. We do have evidence that some students are aware of limitations of examples in a proof context (e.g., Weber 2010) and that students can work productively and strategically with examples, including using examples as a source of generalization (e.g., Ellis et al. 2012). However, we also identified some traits in mathematicians' proving activity that are less typical in our understanding of students' proof practices according to the literature (e.g., Stylianides and Stylianides 2009; Zaslavsky et al. 2012). In particular, we identified three traits that were key in mathematicians' success with leveraging examples to prove. First, we saw the mathematicians display metacognitive awareness in their choice and use of examples. Throughout many of the survey and interview responses, it was clear that the mathematicians were always aware of their overall proving activity, and in particular they understood the role that their example-related activity played in their broader mathematical work. Second, our results also contribute to an understanding of the nature of the back and forth relationship between proof (and examples) and disproof (and counterexamples) for expert provers. It is not the case that mathematicians set out to prove and engage in a neat, clean, logical, abstract, algebraic process proceeding from axioms to deductions. Instead, they make missteps and they abandon false routes. Although this is not a brand new insight, our data reveals specifics about such non-linear proving (especially the relationship between developing examples and counterexamples). These insights about this back and forth relationship can be valuable in helping us make sense of the ways in which mathematicians make progress towards a proof, highlighting the complex, nonlinear nature of proving, even for expert provers. Third, the mathematicians displayed persistence and self-confidence in their work, viewing impasses in a proof as an opportunity to learn rather than an excuse to give up. This selfconfidence may be a possible factor contributing to a mathematician's attention to the back and forth relationship, allowing him or her to embrace being stuck in ways that may make students uncomfortable.

Our findings from these key traits suggest four pedagogical implications for helping students more effectively leverage examples in their proof activity:

1. Do not discourage or denigrate example use. We acknowledge that it is important to help students understand that examples are not suitable substitutes for a proof of a universally true statement, but by high school and especially college, there is evidence that students do indeed understand limitations of example use (e.g., 
Weber 2010). Our findings build on the literature by showing that expert provers do not eschew examples, but in fact they work with them and build understanding from them in specific, strategic ways. Rather than simply emphasizing that examples do not constitute proof, we instead need to help students learn ways to build bridges between the conviction and understanding that can come with examples and deductive argumentation.

2. Encourage and foster deliberate awareness and discussion of example use. We observed a noticeable degree of metacognitive awareness among the mathematicians, and they regularly demonstrated a mindfulness of their work and progress, including a cognizance of how their search for and use of examples was closely connected to their proving activity. Although this phenomenon is perhaps not surprising among experts, an implication could be that fostering metacognitive awareness as we support students' proof activity could be efficacious. Helping students become more aware of their mathematical work and thinking is not a straightforward task, and researchers have previously demonstrated the importance and the challenge of fostering metacognition (Schoenfeld 1979, 1985). Our study lends another voice to work on metacognition, providing further evidence for its value. More research is needed that investigates practices that could help students be more metacognitive in general, and, specifically, more aware of their selection and use of examples in the proving process. We do, however, have some practical ideas. Often what we saw in the mathematicians' interview data is that it was one particular example that supported a key insight (such as Dr. Aldridge's work on Conjecture $4 \mathrm{~b}$ ). When those moments happen in students' proving activity, instructors might stop and draw attention to the ways in which an example afforded the insight. We hypothesize that possible ways to foster metacognition with example use in particular is to engage in activities such as: a) make explicit the example choices one uses in making sense of a conjecture, and discuss with students their affordances and drawbacks; b) emphasize the role of generic examples, how they differ from proof but act as a bridge to a broader deductive argument; c) ask students to answer reflective questions about their example use when formulating, making sense of, and proving conjectures; d) make example choice explicit when students share their ideas more broadly with the class, and open up example choice and use to a public discussion; e) reinforce the link between a particular example and a moment of insight.

3. Explicitly highlight example use in proving. In instruction, we should expose students to different kinds of examples in different domains and be explicit with them about the roles they play in relation to particular conjectures. We saw in our data that mathematicians used some simple examples merely to check the reasonability of a conjecture, while some used extreme examples to test the limits of a conjecture, and still others used examples to gain key insights about a proof. Students might benefit from being exposed to various kind of examples in particular mathematical domains, and instructors could be explicit with them about different types of examples that are available and the roles they play in relation to certain conjectures. For example, such activity might include having students answer reflective questions about their example choices as they explore a conjecture, or teaching them how a generic example differs from, but can lead to, a valid proof. A stronger understanding of the strategies mathematicians employ as they 
use examples to develop, explore, and prove conjectures may ultimately inform the design of instructional practices and curricula that effectively foster students' abilities to prove. More research is needed to investigate such practices and interventions, and we are engaging in such work moving forward.

4. More broadly, shift norms about what it means to be a competent or successful prover. Our findings highlight the importance of shifting the norms for both mathematical activity and proving activity in our instruction. People who are successful at mathematics - in fact, the most successful people, professional mathematicians themselves experience struggles, and this is a normal, natural, and expected aspect of doing mathematics (and of proving). Thus, we should strive to emphasize the truth about mathematicians' processes and normalize it in our instruction and in the development of the classroom cultures we form in instruction. In most undergraduate proof courses, the model of instruction tends to be the presentation of alreadycomplete, beautiful proofs as exemplars. Seldom do we share proofs in progress with students, the challenges in developing proofs, and other evidence that can normalize this back-and-forth behavior. We cannot expect students to develop this kind of persistence if we do not model it ourselves. Thus, one potential pedagogical implication is that we should share these kinds of struggles and present in-progress proof activities with undergraduates in proof courses as part of an explicit attempt to change the culture of what it means to be successful mathematically.

Mathematicians possess an awareness of the powerful role examples can play in exploring, understanding, and proving conjectures, as well as the ability to implement example-related activity in meaningful ways. Yet, the role examples play in proof-related activities in mathematics classrooms, secondary school classrooms, as well as undergraduate classrooms, often stands in stark contrast to the role examples play in the proof-related activities of mathematicians. Our findings build on the literature and suggest important ways in which thinking with examples does indeed serve as a critical step towards proof, and we have offered some implications to help students learn to think critically about how they can draw upon examples as they engage in exploring and proving conjectures. More work remains to be done, however, in translating findings such as these into concrete ways to help students leverage examples effectively.

Acknowledgments The research described in this paper is supported by the National Science Foundation (DRL-0814710 and DRL-1220623). The views expressed do not reflect official positions of the NSF. The authors wish to thank the following people for their contributions to these projects: Tracy Carolan, Jenny Cooper, Fatih Dogan, Chuck Kalish, Eric Knuth, Bukky Leonard, Zekiye Ozgur, Rebecca Vinsonhaler, Candace Walkington, Caro Williams-Pierce, and Orit Zaslavsky, as well as the editors and anonymous reviewers for their feedback on the paper.

\section{References}

Alcock, L. J., \& Inglis, M. (2008). Doctoral students' use of examples in evaluating and proving conjectures. Educational Studies in Mathematics, 69, 111-129.

Andreescu, T., Andrica, D., \& Feng, Z. (2007). 104 number theory problems: From the training of the USA IMO team. Boston, MA: Birkhauser. 
Antonini, S. (2003). Non-examples and proof by contradiction. In N. A. Pateman, B. J. Dougherty, \& J. Zilliox (Eds.), Proceedings of the 27th Conference of the International Group for the Psychology of Mathematics Education (vol. 2, pp. 49-56).

Antonini, S. (2006). Graduate students' processes in generating examples of mathematical objects. In J. Novotna, H. Moraova, M. Kratka, \& N. Stehlikova (Eds.), Proceedings of the 30th Conference of the International Group for the Psychology of Mathematics Education (vol. 2, pp. 57-64).

Balacheff, N. (1987). Processus de prevue et situations de validation. Educational Studies in Mathematics, 18(2), 147-176.

Ball, D. L., Hoyles, C., Jahnke, H. N., \& Movshovitz-Hadar, N. (2002). The teaching of proof. In L. I. Tatsien (Ed.), Proceedings of the international congress of mathematicians, vol. III (pp. 907-920). Beijing: Higher Education.

Bills, L., \& Rowland, T. (1999). Examples, generalization and proof. In L. Brown (Ed.), Making meaning in mathematics. Advances in mathematics education (pp. 103-116). York, UK: QED.

Bills, L., \& Watson, A. (2008). Editorial introduction. Educational Studies in Mathematics, 69, 77-79.

Buchbinder O., \& Zaslavsky O. (2009) A framework for understanding the status of examples in establishing the validity of mathematical statements. In M. Tzekaki, M. Kaldrimidou, \& H. Sakonidis (Eds.), Proceedings of the 33rd Conference of the International Group for the Psychology of Mathematics Education (vol. 2, pp. 225-232).

Buchbinder, O., \& Zaslavsky, O. (2011). Is this a coincidence? The role of examples in fostering a need for proof. ZDM The International Journal on Mathematics Education, 43(2), 269-281.

Carlson, M., \& Bloom, I. (2005). The cyclic nature of problem solving: an emergent multidimensional problem-solving framework. Educational Studies in Mathematics, 58, 45-75.

Chazan, D. (1993). High school geometry students' justification for their views of empirical evidence and mathematical proof. Educational Studies in Mathematics, 24(4), 359-387.

Council of Chief State School Officers. (2010). Common core state standards for mathematics. Washington, DC: Council of Chief State School Officers.

Ellis, A.E., Lockwood, E., Williams, C.C.W., Dogan, M.F., \& Knuth, E. (2012). Middle school students' example use in conjecture exploration and justification. In L.R. Van Zoest, J.J. Lo, \& J.L. Kratky (Eds.), Proceedings of the 34th Annual Meeting of the North American Chapter of the Psychology of Mathematics Education. Kalamazoo, MI.

Epstein, D., \& Levy, S. (1995). Experimentation and proof in mathematics. Notice of the AMS, 42(6), 670674.

Goldenberg, P., \& Mason, J. (2008). Spreading light on and with example spaces. Educational Studies in Mathematics, 69(2), 183-194.

Harel, G. (2001). The development of mathematical induction as a proof scheme: A model for DNR-based instruction. In S. Campbell \& R. Zaskis (Eds.), Learning and teaching number theory, Journal of Mathematical Behavior (pp. 185-212). Westport, CT: Albex Publishers.

Harel, G. (2008). What is mathematics? A pedagogical answer to a philosophical question. In R.B. Gold \& R. Simons (Eds.), Current issues in the philosophy of mathematics from the perspective of mathematicians. Washington, DC: Mathematical American Association.

Harel, G., \& Sowder, L. (1998). Students' proof schemes: results from exploratory studies. Issues in Mathematics Education, 7, 234-283.

Healy, L., \& Hoyles, C. (2000). A study of proof conceptions in algebra. Journal for Research in Mathematics Education, 31(4), 396-428.

Iannone, P., Inglis, M., Mejia-Ramos, J. P., Simpson, A., \& Weber, K. (2011). Does generating examples aid proof production? Educational Studies in Mathematics, 77, 1-14.

Kloosterman, P., \& Lester, F. (2004). Results and interpretations of the 1990 through 2000 mathematics assessments of the National Assessment of Educational Progress. Reston, VA: National Council of Teachers of Mathematics.

Knuth, E. (2002a). Proof as a tool for learning mathematics. Mathematics Teacher, 95(7), 486-490.

Knuth, E. (2002b). Secondary school mathematics teachers' conceptions of proof. Journal for Research in Mathematics Education, 33(5), 379-405.

Knuth, E., Choppin, J., \& Bieda, K. (2009). Middle school students' production of mathematical justifications. In D. Stylianou, M. Blanton, \& E. Knuth (Eds.), Teaching and learning proof across the grades: A K-16 perspective (pp. 153-170). New York, NY: Routledge.

Mason, J., \& Watson, A. (2008). Mathematics as a constructive activity: Exploiting dimensions of possible variation. In M. Carlson \& C. Rasmussen (Eds.), Making the connection: Research and practice in undergraduate mathematics (pp. 189-202). Washington, DC: MAA.

Michener, E. R. (1978). Understanding understanding mathematics. Cognitive Science, 2, 361-383. 
National Council of Teachers of Mathematics. (2000). Principles and standards for school mathematics. Reston, VA: Author.

Pedemonte, B., \& Buchbinder, O. (2012). Examining the role of examples in proving processes through a cognitive lens: the case of triangular numbers. ZDM The International Journal on Mathematics Education, 43(2), 257-267. doi:10.1007/s11858-011-0311-z.

Porteous, K. (1990). What do children really believe? Educational Studies in Mathematics, 21, 589-598.

Rittle-Johnson, B., Star, J. R., \& Durkin, K. (2009). The importance of familiarity when comparing examples: impact on conceptual and procedural knowledge of equation solving. Journal of Educational Psychology, 101(4), 836-852.

Rowland, T. (2001). Generic proofs in number theory. In S. Campbell \& R. Zazkis (Eds.), Learning and teaching number theory: Research in cognition and instruction (pp. 157-184). Westport, CT: Ablex Publishers.

Sandefur, J., Mason, J., Stylianides, G. J., \& Watson, A. (2013). Generating and using examples in the proving process. Educational Studies in Mathematics. doi:10.1007/s10649-012-9459-x.

Savic, M. (2012). What do mathematicians do when they have a proving impasse? In The Electronic Proceedings for the Fifteenth Special Interest Group of the MAA on Research on Undergraduate Mathematics Education. February 23-25. Portland, OR: Portland State University.

Schoenfeld, A. (1979). Explicit heuristic training as a variable in problem-solving performance. Journal for Research in Mathematics Education, 10(3), 173-187.

Schoenfeld, A. H. (1985). Mathematical problem solving. Orlando, FL: Academic Press, Inc.

Schoenfeld, A. H. (1992). Learning to think mathematically: Problem solving, metacognition and sense making in mathematics. In D. Grouws (Ed.), Handbook of research on mathematics teaching and learning (pp. 334-370). New York, NY: Macmillan.

Simon, M. A., \& Blume, G. (1996). Justification in the mathematics classroom: a study of prospective elementary teachers. Journal of Mathematical Behavior, 15, 3-31.

Sowder, L., \& Harel, G. (1998). Types of students' justifications. Mathematics Teacher, 91, 670-675.

Strauss, A., \& Corbin, J. (1998). Basics of qualitative research: Techniques and procedures for developing grounded theory (2nd ed.). Thousand Oaks, CA: Sage Publications, Inc.

Stylianides, G., \& Stylianides, J. (2009). Facilitating the transition from empirical arguments to proof. Journal for Research in Mathematics Education, 40(3), 314-352.

Tsamir, P., Tirosh, D., \& Levenson, E. (2008). Intuitive non-examples: the case of triangles. Educational Studies in Mathematics, 69(2), 81-95.

Watson, A., \& Mason, J. (2005). Mathematics as a constructive activity: Learners generating examples. Mahwah, NJ: Erlbaum.

Watson, A., \& Shipman, S. (2008). Using learner generated examples to introduce new concepts. Educational Studies in Mathematics, 69(2), 97-109. doi:10.1007/s10649-008-9142-4.

Weber, K. (2008). How mathematicians determine if an argument is a valid proof. Journal for Research in Mathematics Education, 39(4), 431-459.

Weber, K. (2010). Mathematics majors' perceptions of conviction, validity, and proof. Mathematical Thinking and Learning, 12, 306-336.

Weber, K., Inglis, M., \& Mejia-Ramos, J. P. (2014). How mathematicians obtain conviction: implications for mathematics instruction and research on epistemic cognition. Educational Psychologist, 49(1), 36-58. doi:10.1080/00461520.2013.865527.

Yackel, E., \& Hanna, G. (2003). Reasoning and proof. In J. Kilpatrick, W. G. Martin, \& D. Schifter (Eds.), $A$ research companion to the principles and standards for school mathematics (pp. 227-236). Reston, VA: NCTM.

Zaslavsky, O., Nickerson, S.D., Stylianides, A. J., Kidron, I., \& Winicki, G. (2012). The need for proof and proving: mathematical and pedagogical perspectives. In G. Hanna \& M. de Villiers (Eds.), Proof and proving in mathematics education: The 19th ICMI Study (New ICMI Study Series, vol. 15). New York, NY: Springer.

Zazkis, R., \& Leikin, R. (2008). Exemplifying definitions: a case of a square. Educational Studies in Mathematics, 69(2), 131-148.

Zodik, I., \& Zaslavsky, O. (2008). Characteristics of teachers' choice of examples in and for the mathematics classroom. Educational Studies in Mathematics, 69(2), 165-182. doi:10.1007/s10649-008-9140-6. 